\title{
Circadian Fluctuations of period Protein Immunoreactivity in the CNS and the Visual System of Drosophila
}

\author{
Danielle M. Zerr, ${ }^{1, a}$ Jeffrey C. Hall, ${ }^{1}$ Michael Rosbash,, ${ }^{1,2}$ and Kathleen K. Siwicki ${ }^{1, b}$ \\ 'Department of Biology and ${ }^{2} H$ oward Hughes Medical Institute, Brandeis University, Waltham, Massachusetts 02254, \\ aPresent address: Temple University Medical School, Philadelphia, Pennsylvania 19122, and \\ bPresent address: Department of Biology, Swarthmore College, Swarthmore, Pennsylvania 19081.
}

\begin{abstract}
When the protein encoded by the period (per) gene, which influences circadian rhythms in Drosophila melanogaster, was labeled with an anti-perantibody in adult flies sectioned at different times of day, regular fluctuations in the intensity of immunoreactivity were observed in cells of the visual system and central brain. These fluctuations persisted in constant darkness. Time courses of the changing levels of staining were altered in the per-short mutant: In light/dark cycles, the phase was earlier than in wild-type, and in constant darkness the period was shorter. In a per-long mutant and in behaviorally subnormal germline transformants (involving transduced per DNA), staining intensities were much fainter than in wild-type. Factors involved in initiating or maintaining the per protein cycling were investigated by examining the immunoreactivity in visual system mutants and by exposing wild-type flies to altered light/dark regimes. These genetic and environmental manipulations affected the expression of the per protein in ways that usually parallelled their effects on circadian behaviors.
\end{abstract}

Circadian rhythms are endogenous biological cycles that continue with a period of about $24 \mathrm{hr}$ in the absence of environmental cues. Externally mediated inputs, however, can set thesc cycles in motion and determine the phase of the rhythmicity (reviews: Saunders, 1982; Johnson and Hastings, 1986). The period (per) gene of Drosophila melanogaster is one of the endogenous factors involved in the fly's circadian rhythms. Mutations at this locus-per ${ }^{\mathrm{s}}$, per $\mathrm{r}^{\mathrm{LI}}$, and $p e r^{0 \mathrm{t}}$-shorten, lengthen or effectively abolish the fly's circadian cycles (Konopka and Benzer, 1971; review: Konopka, 1987).

An antibody against the per protein has been used in immunohistochemical assays to localize sites of the gene's expression in embryos, pupae, and adults (Siwicki et al., 1988). Among the most prominent locations of per protein staining in the adult fly are photoreceptor nuclei, putative glial cells in various ganglia, and certain neurons in lateral regions of the central brain.

\footnotetext{
Received Feb. 7, 1990; revised Mar. 29, 1990; accepted Apr. 9, 1990.

We thank Dr. Edward A. Kravitz for use of his microscope; Melanie HamblenCoyle for performing and analyzing the behavioral experiments, as well as for helping with figure preparation; and Lisa Moroz for doing the chromosomal in situ hybridizations. We appreciate comments on the manuscript from Paul E. Hardin and Hildur V. Colot. This work was supported by NIH Grant GM-33205 to M.R. and J.C.II, and by NIII Fellowship NS-07873 to K.K.S.

Correspondence should be addressed to Jeffrey C. Hall, Department of Biology, 235 Bassine Building, Brandeis University, 415 South Street, Waltham, MA 022549110.

Copyright (C) 1990 Society for Neuroscience $0270-6474 / 90 / 082749-14 \$ 03.00 / 0$
}

The intensity of anti-per staining in the visual system was found to be distinctly different at 2 opposite phases of a $24 \mathrm{hr}$ cycle: readily detectable in the middle of the night and essentially absent in the middle of the day (Siwicki et al., 1988). While daily fluctuations in the staining of other cells and tissues were not observed in the original study, they could not be ruled out with data from only 2 timc points.

One objective of the current experiments was to determine if similar staining fluctuations occur in the fly's central brain. Another goal was to compare the temporal modulation of the mutant per and per ${ }^{\mathrm{L}} \mathrm{p}$ proteins to that of the wild-type per gene product, to determine whether such comparisons would correlate with the effects of these mutations on adult behavior. We also assessed the effects of various visual system mutations and manipulations of light/dark cycles on both the basic tissue distribution and the cyclical expression of the per protein. Our principal conclusions follow: (1) There are circadian fluctuations of per protein immunoreactivities in all of the nervous system cell types where they are detectable by the antibody-mediated staining; the periodicity, phase, and strength of a given staining cycle are altered by a variety of genetic variants involving the per gene. (2) Manipulations of light/dark cycles known to influence the fly's circadian behavioral rhythms strongly tend to cxert analogous effects on the fluctuating per protein stainings. (3) Transduction of the light stimuli that initiate and set the phase of these immunoreactivity cycles seems not to involve standard pathways of photoreceptor excitation.

\section{Materials and Methods}

Drosophila strains. The per $^{+}$flies were from either Canton-S (wildtype), yellow white, white tan, or rosy strains. Circadian activity rhythms in these genetically normal or marker-bearing flies are strong, with ca. $24 \mathrm{hr}$ periods (Hamblen et al., 1986; Dushay et al., 1989; and M. Hamblen-Coyle, unpublished observations, for the $w t$ double mutant). Stocks containing period mutations were (1) per $r^{(1)} ; y^{506}$ and per $^{r^{01}} ; A d h^{\text {fn23 }}$ pr $\mathrm{cn}$; the adult locomotor activity of these marked per-zero strains is essentially arrhythmic (e.g., Hamblen et al., 1986); and (2) the per ${ }^{\mathrm{s}}$ and per ${ }^{\llcorner\prime}$ mutants; these had been induced on a Canton-S genetic background and are descendants of the original short- and long-period strains of Konopka and Benzer (1971).

Several types of visual mutants were assayed immunohistochemically: (1) One was an $r d g B$ (retinal-degeneration- $B$ ) mutant, which exhibits light-induced degeneration of certain photoreceptors in the compound eye; mutant $r d g B$ adults were aged in LD conditions for 5 or 19 d posteclosion, before being sectioned between ca. 6 and $11 \mathrm{hr}$ after lights-off [=Zeitgeber Time (ZT) 18-23 (see Figs. 2, 4)]. (2) Adults expressing the norp $A^{\mathrm{P} 24}$, norp $A^{\mathrm{P} 41}$, or nina $E^{\mathrm{O} 17}$ visual-function mutations were sectioned and stained at about the same two $180^{\circ}$ out-of-phase timcs, during LD, as just noted. (3) A disconnected (disco) visual systemdefective/circadian-arrhythmic mutant was studied in LD and in DD 
sectioning/staining experiments similar to those performed for wildtype, i.e., with several time points taken per cycle. We used a stock carrying the disco ${ }^{2}$ allele, flanked on the $X$ chromosome by white and forked markers (see Dushay et al., 1989). Mutant disco individuals, which happened to have one of their eyes connected to their brain (via optic ganglia) and the other eye disconnected (hence, no optic ganglia), were selected before sectioning and staining. These bilaterally normal/ mutant flies were detected optically as in Dushay et al. (1989).

Certain germ-line transformants, involving DNA cloned from the per locus, were assayed immunohistochemically: (1) The 14.6 and $8.0 \mathrm{~kb}$ per-locus DNA fragments had been previously transformed into per $^{01} ; A d h^{\text {fn23 }} p r$ cn flies, as described by Zehring et al. (1984); these transduced per ${ }^{+}$inserts also carry an $A d h^{+}$allele (" $\mathrm{fn} 23$ " being null for alcohol dehydrogenase activity); transformed per ${ }^{01} ; 14.6: 21 / \operatorname{In}(2 L R) O$, $C y A d h^{\mathrm{nB}}$ and $p e r^{\mathrm{N1}} ; 8.0: 4 / \operatorname{In}(2 L R) O, C y A d h^{\mathrm{nB}}$ flics werc crossed to per ${ }^{01} ; A d h^{\text {fn } 23}$ pr cn homozygotes. [The numbers after the colons designate 2 particular strains of these transformant types (see Zehring et al., 1984); the $\operatorname{In}(2 L R) O$ balancer, carrying a $C u r l y(C y)$ wing mutation, is hereafter called $C y O$.] From the non-Cy progeny, per ${ }^{01} ; 14.6: 21$ (or 8.0:4) $A d h^{\text {fn23 }}$ pr $c n$ flies were verified by ethanol tolerance testing (which selects for $A d h^{+}$, hence per+; see Zehring et al., 1984); the survivors were exposed to light/dark cycles (see below), then their locomotor activity was monitored (see below). (2) The $13.2 \mathrm{~kb} \mathrm{per}{ }^{+}$DNA fragment had been previously transformed into flies whose genetic background is per $; r y^{506}$ (Citri et al., 1987; Yu et al., 1987a; the per $^{+}$inserts here are marked by $r y^{+}$DNA); transformed per ${ }^{01} ; 13.2: 34 / C y O ; r y^{506}$ parents (whose $13.2 \mathrm{~kb}$ per $^{+}$insert is on chromosome 2) were crossed to perot; $r y^{506}$; the per ${ }^{01}$; $13.2: 34, r y^{506}$ progeny were selected (as $C y^{+} r y^{+}$flies) then monitored bchaviorally (scc bclow). Flics carrying 3 copics of transduced per-locus DNA fragments $(y w ; 13.2: 34 / C y O ; 13.2: 2 / 13.2: 2)$, were constructed by genetic crosses using 2nd- and 3rd-chromosomal balancers (strain 13.2:2 has a per ${ }^{+}$insert on chromosome 3 ); it was confirmed that this stock had 3 transduced "doses" of per ${ }^{+}$, by in situ hybridization of per DNA probes to salivary gland chromosome squashes, before these transformed flies were used on behavioral and histochemical experiments.

Light/dark regimes. Drosophila were exposed to various different conditions of light and/or darkness before they were sectioned and stained. All of the following experiments were carried out at $25^{\circ} \mathrm{C}$. Flies were reared on a cornmeal/molasses/yeast/agar/"Tegosept" medium (the latter ingredient being a mold inhibitor). Exposure of adults to a given light /dark (or other) regime (see below) was effected by putting the flies into food-containing glass vials (usually 2-5 animals/vial), which were in a constant-temperature $\left(25^{\circ} \mathrm{C}\right)$ incubator whose lights were programmed as follows: (1) "Entrained" flies were raised in $12 \mathrm{hr}$ light/12 hr dark (12/12 LD) cycles, then - as adults - exposed to 3-7 further LD cycles before being frozen for sectioning. (2) "Free-running" flies had been raised and entrained as above (1), then were transferred to constant dark (DD) conditions during the D phase of the final LD cycle; the transfer involved putting the food vials containing LD-exposed flies into light-tight cans and returning them to the $25^{\circ} \mathrm{C}$ incubator, whose LD cycles were switched off; in both the LD and LD $\rightarrow$ DD experiments, flies were usually sectioned at $2-4 \mathrm{hr}$ time points throughout the "assay window," which consisted of at least one 24-hr cycle. [Exceptions: Experiments involving certain of the visual mutants involved only 2 time points per LD cycle (see above).] (3) In "entrainment/constant light" experiments, wild-type flies were put through LD cycles, as in (1) above, and subsequently transferred to "LL" conditions during the L phase of the final LD cycle. (4) "Constant condition" animals, raised in LL or $\mathrm{DD}$, were placed in one of these conditions as 1- to 2-hr-old embryos; for $\mathrm{LL}$, the culture and fly-containing vials were exposed to incandescent white light whose intensity was ca. 160 foot-candles; DD rearing was effected by wrapping vial cultures in aluminum foil and placing them in light-tight cans; these LL or DD cultures remained in LL or DD for 15-22 d; 5-20 adults emerged per culture (after the $10 \mathrm{~d}$ developmental period at $25^{\circ} \mathrm{C}$ ), and these were left undisturbed in such vials, posteclosion; the adults were therefore $5-12 \mathrm{~d}$ old at the time they were frozen and had their per immunoreactivities determined. (5) "Single transition" flies were first raised in LL from 1- to 2-hr-old embryos, as above (4); 15-17 d later, the resulting adults were transferred to DD, as in (2) above. (6) "Pulsed" flies were first raised in DD, as above (4); 15-17 d later, the flies were subjected to one $12 \mathrm{hr}$ period of light (intensity ca. 160 foot-candles), after which they were placed back into DD, as in (2) above.

Circadian rhythm monitoring. Transformed flies (see above) usually had their circadian locomotor activity rhythms monitored prior to being assayed immunohistochemically. These behavioral tests were performed by first entraining the adults in $12 / 12 \mathrm{LD}$ cycles; then the flies were put into constant darkness (in $25^{\circ} \mathrm{C}$ incubators), where activity events were counted automatically as described by Hamblen et al. (1986). After determination of their free-running locomotor behavior, the transformants were removed from the monitoring devices, put individually into food vials, then placed back into $L D$ for 3-5 d before sectioning and staining. Other behavioral tests were performed using wild-type flies that were not subsequently assayed immunohistochemically. In these experiments, LL-reared adults (with the light intensity as specified above) were monitored for locomotor activity either in continuing conditions of constant light or after a transition to constant darkness. "LL" for this behavioral testing was fluorescent light whose intensity ranged from ca. 40 to 65 foot-candles, at different locations within the incubator containing the monitors.

Immunohistochemical assays. Expression of the per gene product was assayed in tissue sections, using an antibody - called "anti-S"-raised in a rabbit by Siwicki et al. (1988). Frozen flies were sectioned at 10 $\mu \mathrm{m}$, in the horizontal plane, and stained with affinity-purified anti-S antibody and a Vectastain ABC immunoperoxidase kit (Vector Labs), as described by Siwicki et al. (1988).

Scoring of immunoreactivity intensities. The stained and mounted sections were coded (see below), then viewed with Nomarski optics at $160 \times$. Levels of staining were subjectively scored using an intensity scale of $0-4$, in increments of 0.5 . Fly identities and times of freezing were decoded only after the scoring was completed. An exception involved scoring of stained disco sections; the genotype of these flies was apparent by inspection (see legend to Fig. 7), so that scoring for this mutant was blind only with respect to times of sectioning. The 2 scores for each of the 3 cell types in each fly were averaged (except in the case of Table 2, where the data came from blind scoring by one investigator only). In some of the experiments (e.g., Figs. 3-5), the plotted points represent the mean scores $( \pm \mathrm{SEM}$ ) of several flies of a given genotype at a given time point. For these, the averages of the 2 investigators' scores was first computed for the cell type in question, followed by calculating a mean ( \pm SEM) for all the animals sectioned at the same time (i.e., a mean of a mean).

\section{Results}

Daily cycling of the per protein in wild-type and per mutants

The per protein in the nervous system of $D$. melanogaster adults entrained in 12/12 LD cycles exhibited daily fluctuations in the intensity of this gene's expression, detected immunohistochemically by application of the "anti-S" reagent. This antibody is specific for the per protein (Siwicki et al., 1988). The immunogen used to produce anti-S was a synthetic peptide (14mer), called "peptide-S"' because it occurs in the per polypeptide near the site of the pers mutation (cf. Baylies et al., 1987; Yu et al., 1987b).

Figure 1 shows the approximate peaks and troughs of staining for the per protein in the 3 cell types that were immunoreactive: photoreceptors, nerve cell bodies in lateral regions of the central brain cortex, and glial cells. These temporally dependent differences in per protein immunoreactivity had been previously observed in the photoreceptors and optic lobe glial cells by Siwicki et al. (1988); that report also presented evidence on the nuclear nature of the photoreceptor staining.

In the more detailed studies reported here, stainings of neurons and putative glia in the central brain were found to cycle as well. The relevant neuronal cell bodies are shown in Figure $1, A, C$. The signals in these perikarya have routinely appeared to be cytoplasmic (see Siwicki et al., 1988), though this has been determined only at low resolution. The tentative identification of the small stained cells at the edges of neuropil regions as glial (Fig. $1 A$ ) is based on their similar positions to those of neuropil glia in other insects (Wigglesworth, 1959; Hoyle, 1986; and see Siwicki et al., 1988, for further discussion).

The fluctuations in staining intensity were determined by mi- 


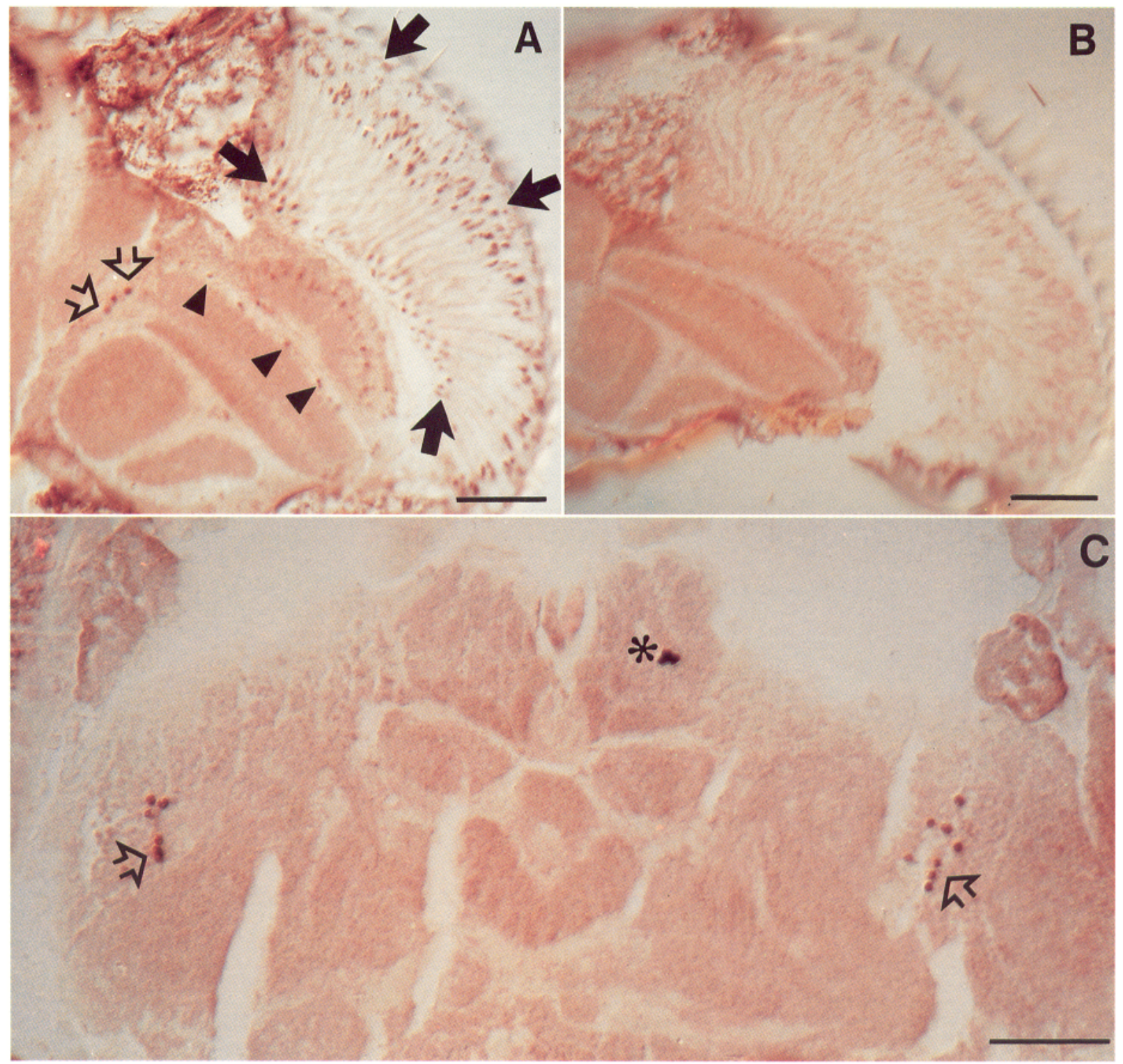

Figure 1. Staining of the per protein in the heads of wild-type flies exposed to light/dark cycles. Horizontal sections of the heads of wild-type flies were stained with the anti-per antibody. After $7 \mathrm{~d}$ in $12 \mathrm{hr}$ light $/ 12 \mathrm{hr}$ dark cycles $(12 / 12 \mathrm{LD})$, the 2 flies depicted in $A$ and $B$ were sectioned and stained at opposite phases of the LD cycle. Lights were on from 0000 to 1200, and off from 1200 to 2400 [see Zeitgeber Time (ZT) bar in Fig. 2]. $A$, Late in the night (in this specific fly, ZT = 1930), staining in the photoreceptors (filled arrows), glia (arrowheads), and lateral neurons (open arrows) is strongest. $B$, Late in the day ( $\mathrm{ZT}=0950$ in this case), there is no evidence of staining. $C$, The brain neurons (arrows) expressing the per protein are clustered in a region of cell body-containing cortex, lateral to the protocerebral neuropil and medial to the optic lobes. There are approximately 15-20 stained neurons on each side of the brain, about half of them being in this section. This fly was sectioned late in the night (ZT 2230). The material marked by the asterisk is debris (not additional per-expressing neurons). Scale bars, $50 \mu \mathrm{m}$.

croscope observations and subjective scorings, which were performed as follows: The basic immunohistochemical procedures for anti-S-mediated staining of the per protein were carried out according to the detailed description of these methods in Siwicki et al. (1988). These standarized conditions were adhered to as closely as possible in every experiment. Furthermore, to verify the consistency of these staining results and to allow comparisons to be drawn among data from different experiments, at least 2 wild-type flies were sectioned during times of peak staining intensities (i.e., late dark phase through early light), as controls accompanying tests of the effects of a given genetic variant or environmental manipulation. To quantify the variable degrees of immunoreactivities, a score of " 4 " was assigned to the highest intensity observed in a given tissue. A score of "0" means that no staining was detectable above the level of background reaction observed in the per ${ }^{01}$ mutant. This arrhythmic per variant, caused by a nonsense mutation located upstream of peptide-S (Baylies et al., 1987; Yu et al., 1987b), provided a genetic control for the specificity of the antibody (Siwicki et al., 1988). The intensities of staining in each fly were scored blindly and independently by 2 investigators. After examination of the sections from a given fly, separate scores were assigned for the 3 per-expressing cell types in the adult head (see above).

Figure 2 shows the time course of daily changes in per protein staining in wild-type flies exposed to LD. Staining was most intense at the end of the dark phase of the LD cycle and began 


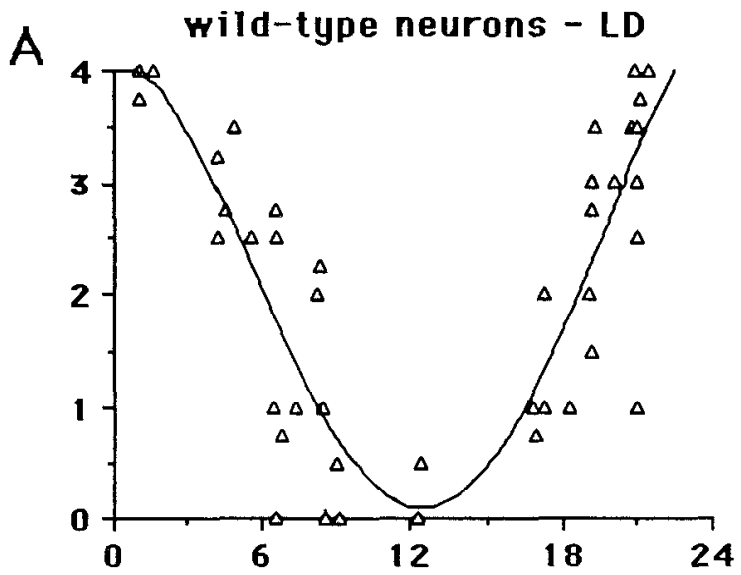

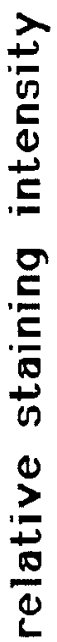
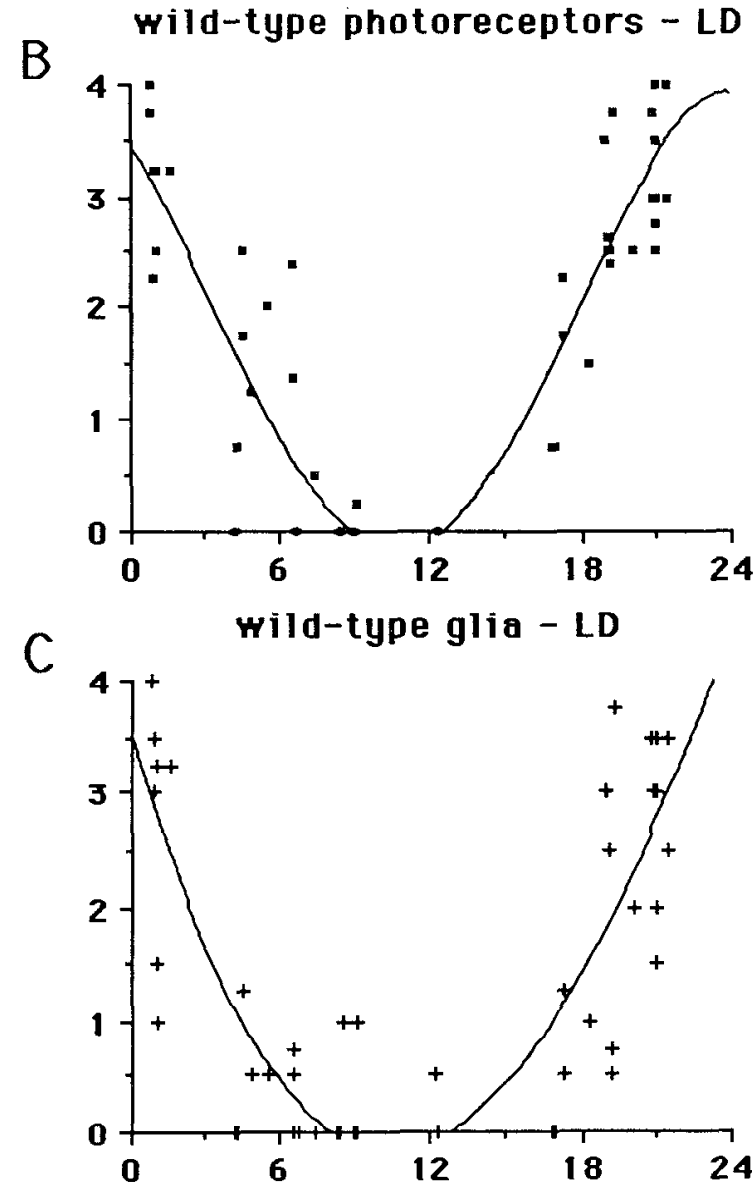

Zeitgeber time

Figure 2. LD cycling of the per protein in wild-type adults. Levels of immunoreactivities in the lateral neurons $(A)$, photoreceptors $(B)$, and glia $(C)$, in $\mathrm{per}^{+}$flies, were quantified by determining staining intensities (ordinates). The flies had been entrained for 3-7 d in $12 \mathrm{hr}$ light $/ 12 \mathrm{hr}$ dark (LD) cycles before they were sectioned at $2-4 \mathrm{hr}$ intervals, during a final LD cycle. This cycle is designated in Zeitgeber Time (ZT), for which $0000-1200 \mathrm{hr}$ is the light phase (open portion of bar below the abscissa) and 1200-2400 is dark (filled portion of bar). The intensity values were arrived at by observing stained sections through the microscope and subjectively specifying, on a scale of $0-4$, the level of immunoreactivity in a given cell type; these assignments were done blind (see Materials and Methods). The points on these 3 graphs represent the averages of scorcs assigncd by 2 investigators, for 58 animals for 8 separate experiments. Several of the plotted values on each graph to decrease after the lights came on. The immunoreactivity continued to diminish throughout the day and was undetectable by the end of the light phase. Staining was detected again starting about $2 \mathrm{hr}$ after lights went off and increased in intensity through the night.

While the time courses of increasing staining intensity during the night were virtually identical for all 3 cell types, the rates of decay of the staining during the day appear to differ. Specifically, staining in photoreceptors and glial cells (Fig. 2B, C) decayed faster during the day than did neuronal staining (Fig. $2 A)$. Indeed, several individual animals sectioned during the day were notable for exhibiting no staining in the visual system, though their neurons were still reactive. These differences account for the fact that we did not observe cycling in the lateral brain neurons in our earlier study (Siwicki et al., 1988), since those data were collected from only 2 time points: the middle of the day and the middle of the night. As illustrated in Figure 2 , there is, overall, a noticeable difference in the intensity of photoreceptor staining between 0600 and 1800 (nominally a 3 -fold greater score at the latter time), whereas the intensity of neuronal staining at these 2 time points is rather similar (nominally a score of " 2 " at each phase).

The anti-S antibody detects per-specific signals in the adult thorax (Siwicki et al., 1988): scattered cells (which may be glia) in the ventral ganglia and many cells in the gut (Liu et al., 1988). Although the intensity of thoracic immunoreactivity was not quantified systematically in the current study, it appeared as if the levels of staining in the ventral ganglia cycled in phase with that in the brain, whereas such intensities did not fluctuate in the gut (data not shown).

The time courses of per protein staining in the central and peripheral nervous system of the head were different from wildtype in flies expressing mutant per alleles. In per ${ }^{L 1}$ (Fig. 3), the staining in LD was less intense than in wild-type. The time course (Fig. 3C) shows an apparently low-amplitude cycling of per. $^{\text {I. }}$ protein staining. The putative cycles in this mutant were so "gentle" that it is difficult to compare the shapes and peak times of these curves to those determined for per $^{+}$(Fig. 2 vs Fig. 3C).

The peak staining intensity of the pers protein was comparable to that of per (Fig. 4). Yet several differences were observed between the mutant and normal time courses (Fig. 4). For example, in the brain neurons, the staining of the pers protein decayed sooner than the per $^{+}$protein during the day (Fig. 4A). The trough for the mutant's neuronal immunoreactivity was about $4 \mathrm{hr}$ earlier than in wild-type. In the photoreceptors and glia, staining of the pers protein peaked earlier in the night, compared to wild-type, and began to decline well before the lights came on (Fig. 4, B, C). Also, in the photoreceptors, the rise of the staining intensity during the night occurred somewhat faster in pers than in per ${ }^{+}$(Fig. 4B). These pers-induced alterations in the phases of the protein cyclings were reproducible, in that similar time courses of fluctuations in pers protein stainings were obtained in 3 additional LD experiments - one that

\footnotetext{
represent 2-3 overlapping data points. In addition, there are only 56 points in $A$ (vs 58 in $B$ and $C$ ) because, for 2 of the flies, the quality of the particular sections at the plane of the lateral neurons' location did not permit their staining intensities to be scored (also see legend to Table 2). The curves werc drawn based on softwarc-aided fittings of the plotted points to polynomial functions.
} 

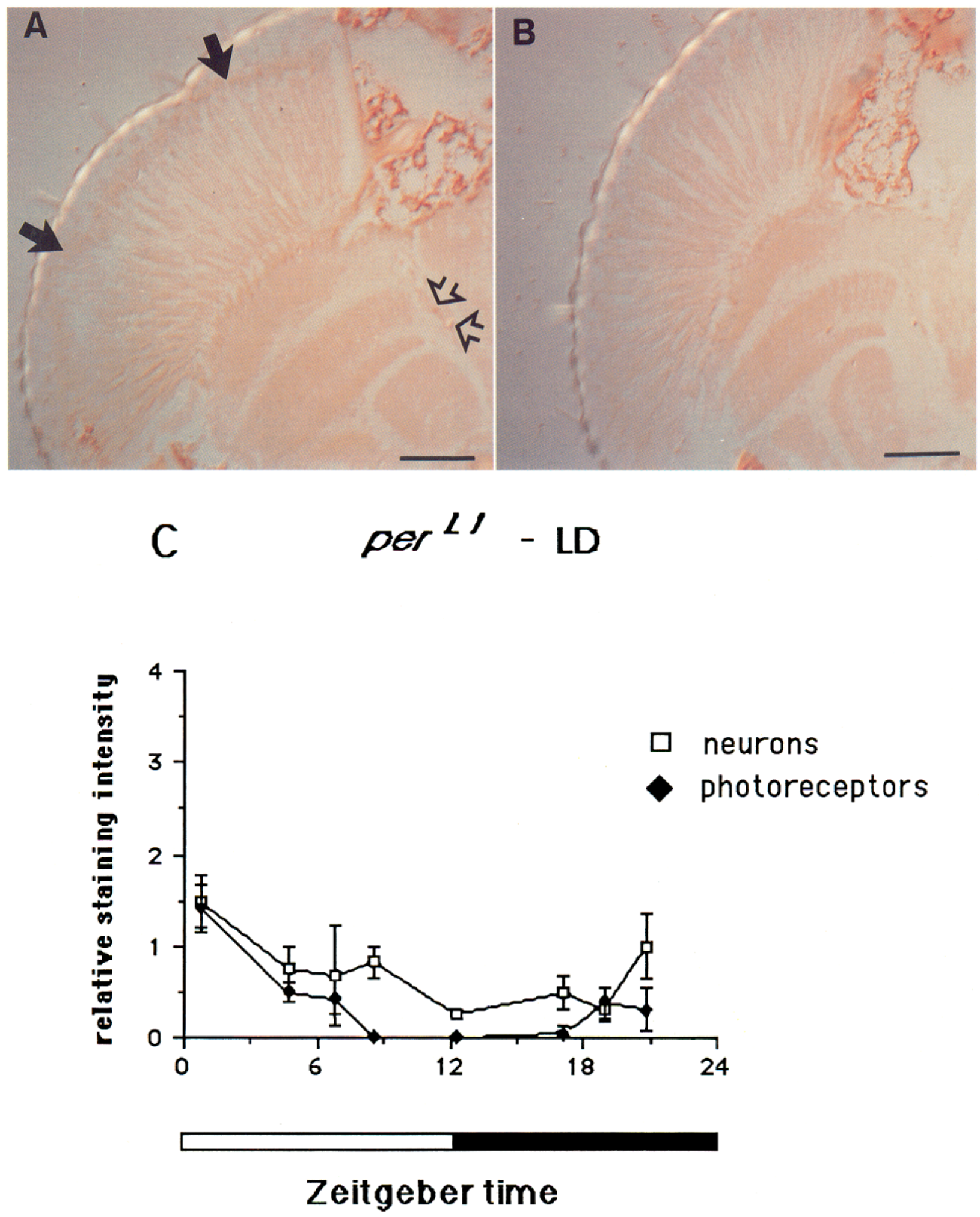

Figure 3. Low-amplitude cycling of the per ${ }^{\mathrm{L} 1}$ protein in LD. Mutant per ${ }^{\mathrm{L} 1}$ flies were sectioned and stained after $7 \mathrm{~d}$ in $12 / 12 \mathrm{LD}$ cycles. During a final LD cycle, fly $A$ was frozen early in the day (ZT 0140) and $B$ early in the night (ZT 1330). The peak intensity of the per ${ }^{\mathrm{LI}}$ protein staining is exemplified in $A$ by the rather faint labeling of photoreceptor nuclei (filled arrows) and brain neurons (open arrows); no glial immunoreactivity was detectable in this fly. $B$ represents the trough of staining for this mutant. Scale bars, $50 \mu \mathrm{m}$. $C$, Quantified per ${ }^{\mathrm{Ll}}$ cycling: The plots for the neuronal and photoreceptor stainings include data from 2 separate experiments, with each time point representing a mean score $( \pm$ SEM) from 4 sectioned flies. The $3 \mathrm{per}^{+}$controls in these experiments, sectioned late in the dark phase or early in the light phase, had mean staining intensities of $3.7 \pm$ 0.1 in lateral neurons and $3.1 \pm 0.3$ in photoreceptor nuclei. Staining scores were determined as described in Materials and Methods. 


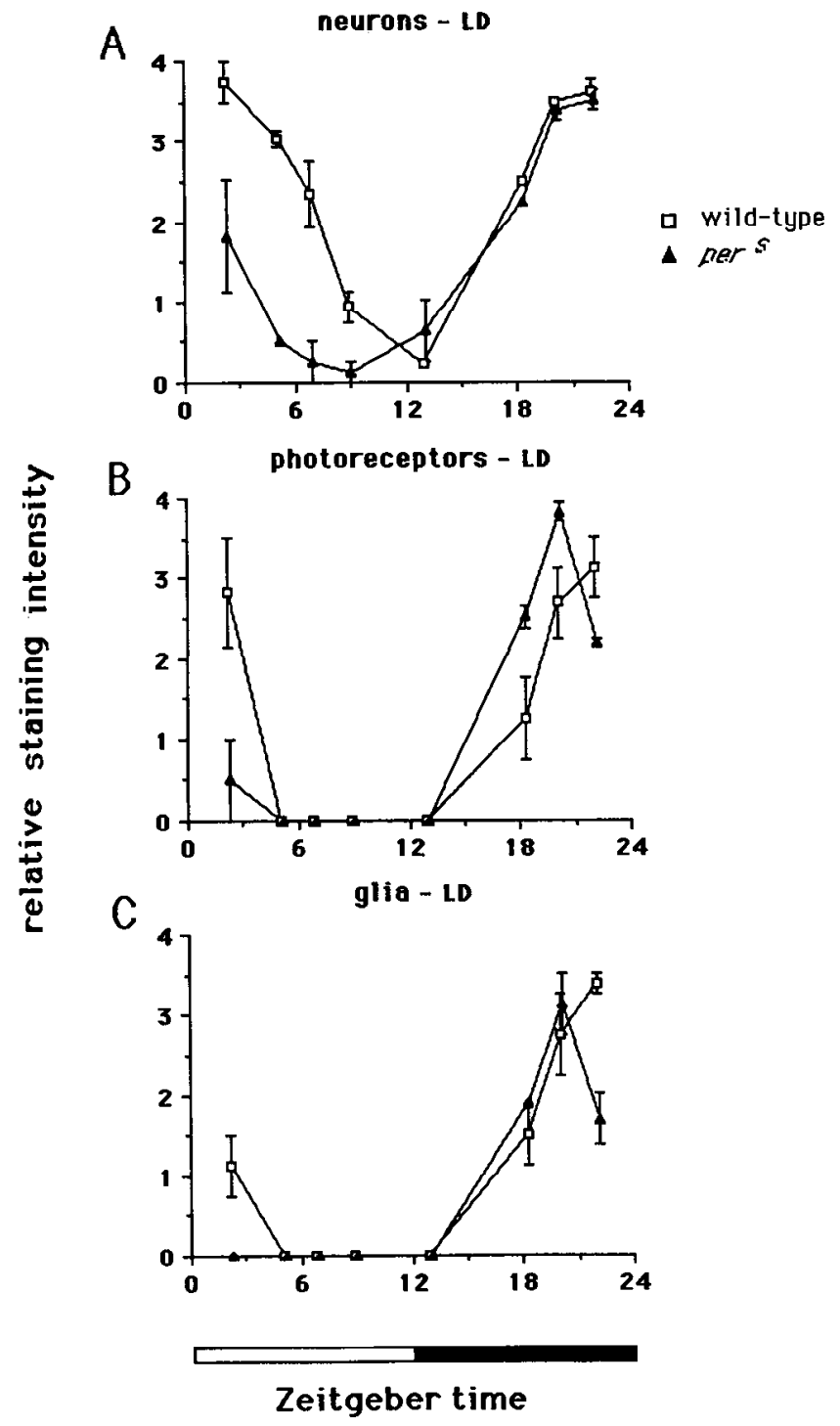

Figure 4. Cycling of the pers protein in LD. Wild-type and mutant $p_{e r}$ flies were exposed to LD for $7 \mathrm{~d}$ before being sectioned and stained during a final LD cycle. In this experiment, 16 mutant and 16 wildtype flies were processed in parallel. The wild-type data are a subset of those plotted in Figure 2. Each point represents the mean ( \pm range) of staining scores from 2 flies. Scores were determined as described in Materials and Methods. The 3 separate graphs represent fluctuations in staining intensities exhibited by $(A)$ lateral brain neurons, $(B)$ photoreceptor nuclei, and $(C)$ central brain plus optic lobe glia.

directly compared mutant to wild-type (as shown here) and 2 involving $p e r$ s only. Even the finer distinctions in question, such as the earlier trough for $\mathrm{per}^{\mathrm{s}}$ neuronal staining (Fig. 4A) and the premature rise for this mutant's photoreceptor immunoreactivity (Fig. $4 B$ ), were evident in the replications of the basic experiment.

In spite of the phase differences just described, both the pers and wild-type proteins fluctuated with the same cycle durations in $\mathrm{LD}$, in that there is one peak and one trough per $12 \mathrm{hr}$ light/ $12 \mathrm{hr}$ dark cycle in the plots shown in Figure 4 . Similarly, both pers and per ${ }^{+}$adults behaviorally entrain to these kinds of LD cycles: Flies expressing cithcr allclc and placed in these conditions exhibit $24 \mathrm{hr}$ periodicities in their locomotor activity (M. Hamblen-Coyle and D. A. Wheeler, unpublished observations).

In constant darkness (DD), the free-running behavioral cir-

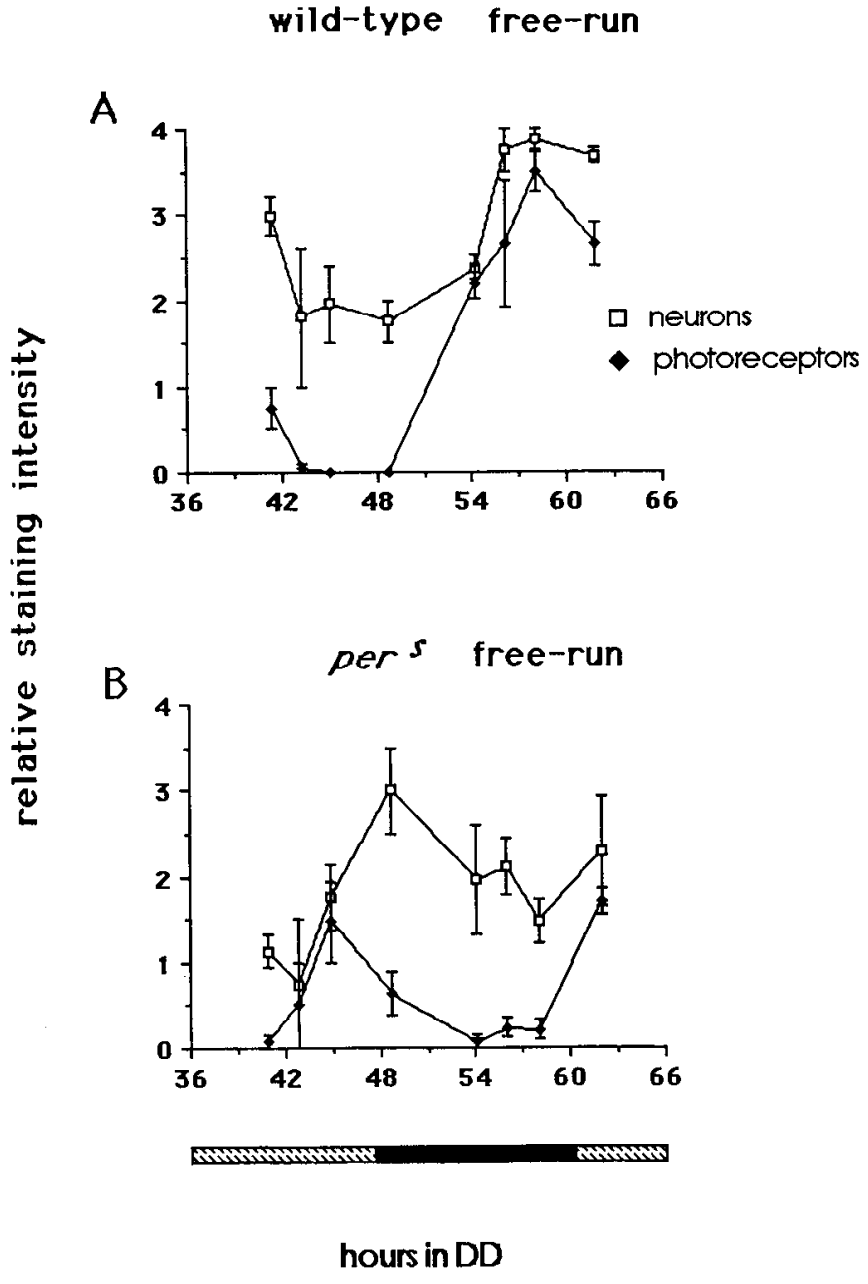

Figure 5. Free-running fluctuations of $\mathrm{per}^{+}$and $\mathrm{per}^{\mathrm{s}}$ protein stainings in constant darkness. After $6 \mathrm{~d}$ in LD, flies were transferred into constant darkness (DD) at ZT 1200 of the last LD cycle and remained in DD for 40-60 hr before sectioning. Thus, time " 0 " on the abscissas (not shown) was the beginning of constant darkness. In lateral brain neurons and in photoreceptor nuclei, staining of the $\operatorname{per}^{+}(A)$ and pers $(B)$ proteins continued to cycle in DD. Glial staining was usually undetectable after $2-3 \mathrm{~d}$ in constant darkness. Each point on the 2 graphs represents the mean score from 2-3 flies. In the horizontal bar at the bottom, the shaded portion represents "subjective day" and the filled portion "subjective night," which correspond to the times when it would have been light or dark, respectively, if the LD cycle had continued.

cadian rhythms of $D$. melanogaster have periods of about 24 $\mathrm{hr}$ in wild-type and about $19 \mathrm{hr}$ in pers flies (e.g., Konopka and Benzer, 1971; Yu et al., 1987b). We monitored free-running fluctuations in the intensity of per protein staining in flies expressing these 2 per alleles. Adults were exposed to LD cycles and then transferred into DD for $40-60 \mathrm{hr}$ before they were sectioned. Both the wild-type and the mutant pers proteins continued to cycle in DD. Figure $5 A$ illustrates the cycling of the per $^{+}$product in constant darkness, showing what we infer to be the second free-running cycle. By extrapolating back to the point where the flies were transferred into $\mathrm{DD}$, the period was calculated to be approximately $24 \mathrm{hr}$, as follows: First, the lowest point on this wild-type was estimated to be ca. $43-48 \mathrm{hr}$ (Fig. $5 A$ ). In $\mathrm{LD}$, the staining troughs occur at the end of the light phases, between ca. ZT 9 and 12 (cf. Fig. 4). If the free-running staining cycles are ca. $24 \mathrm{hr}$ in duration for the wild-type per 


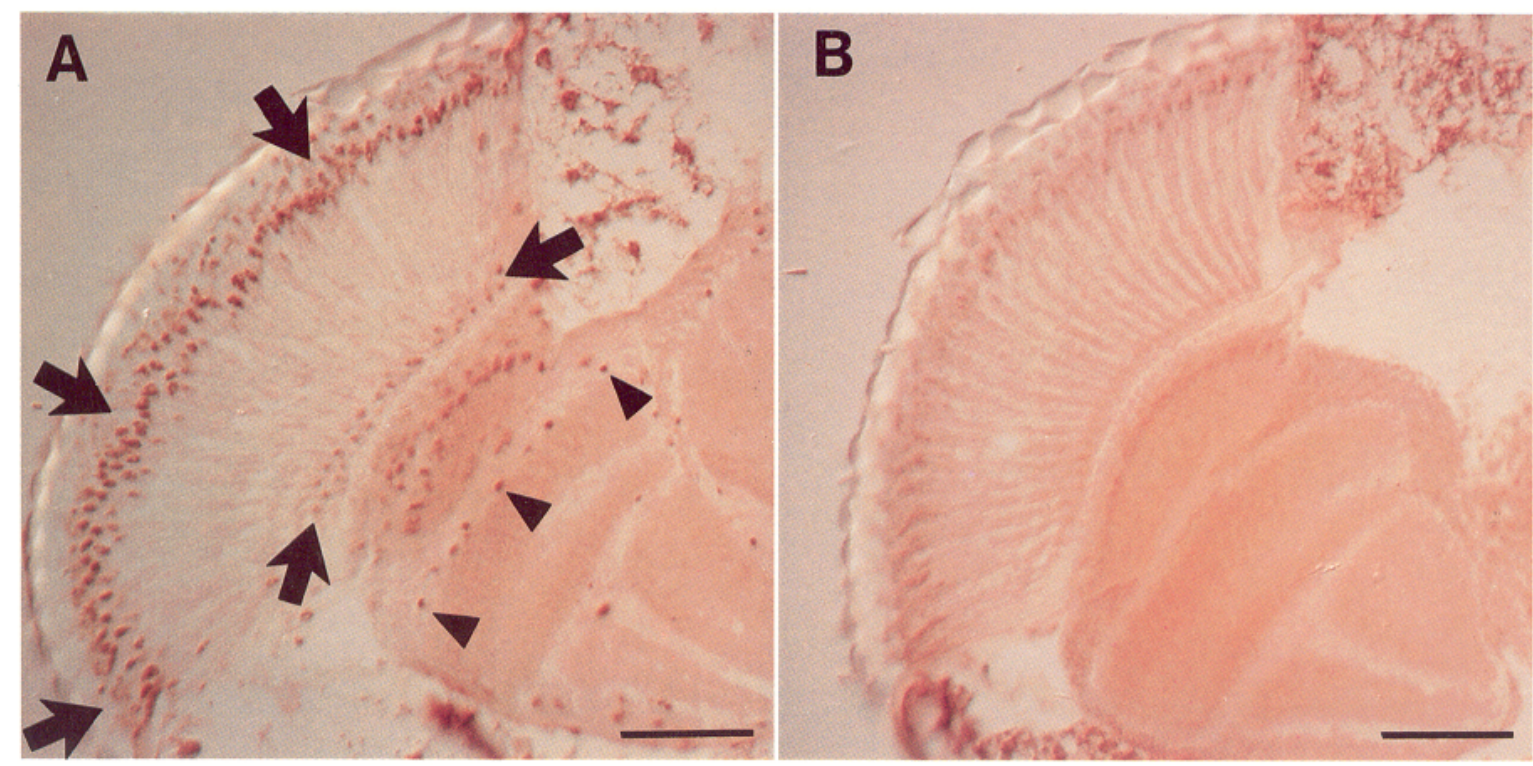

Figure 6. Normal tissue distribution and LD cycling in a blind norpA mutant. Flies expressing the norp $A^{\mathrm{P} 41}$ "no receptor potential" mutation were frozen and sectioned after 4-10 d of entrainment in 12/12 LD. $A$, The fly was frozen during the night at ZT 2045 . $B$, The fly was frozen during the day at ZT 0735. During the night photoreceptor nuclei (arrows), glia (arrowheads), and lateral neurons (not in this plane of section) stained intensely. During the day, only weak photoreceptor staining was detected. Scale bars, $50 \mu \mathrm{m}$.

protein, the first trough in DD (for which no data were collected) would have been between ca. 21-24 hr on the abscissa in Figure $5 A$, and the second should be between ca. $45-48 \mathrm{hr}$, as it was.

Figure $5 B$ shows the staining fluctuation of the $\mathrm{per}^{\mathrm{s}}$ protein, for what appears to be the third cycle in DD. By an extrapolation (similar to that performed for wild-type), the period of this mutant's immunoreactivity cycle was calculated to be approximately $20 \mathrm{hr}$. Thus, the troughs in this mutant's plot (Fig. 5B) were at ca. $40-42 \mathrm{hr}$ then at ca. 54-58 hr. If the free-running pers protein cycles become ca. $5 \mathrm{hr}$ shorter than in wild-type immediately the flies proceed from LD to DD, the first trough (no data collected) would have been between 17-19 hr, the second between 36-38 hr (it was actually a bit later), and the third at ca. 55-57 hr (as did occur). Thus, the free-running periods in Figure $5 B$ are approximately $4 \mathrm{hr}$ shorter than in Figure $5 \mathrm{~A}$.

Since the peak versus trough differences in staining intensities for per $^{\mathrm{L} \text { l }}$ flies were so minimal in LD (Fig. 3), we did not attempt to characterize a free-running staining rhythm for this mutant.

The maximum levels of immunoreactivity for photoreceptors and neurons in DD were generally less than those determined in LD (Figs. 2 and 4 vs Fig. 5). Also, the troughs of the neuronal stainings in DD were never as low as during LD cycles (Figs. 2 and 4 vs Fig. 5). This kind of "DD dampening" has been observed in other biochemical circadian rhythms (e.g., Takahashi et al., 1980; Besharse and Iuvone, 1983). Yet, it must be acknowledged that we are not per se reporting "staining rhythms" for the per protein, in that only about one cycle's worth of immunoreactivity fluctuations was determined in Figure 5.

\section{Visual system mutants}

Mutant $r d g B$ flies were examined to determine whether the per protein staining found in the eye is truly in the photoreceptor cells, as suggested in previous reports (Liu et al., 1988; Saez and Young, 1988; Siwicki et al., 1988). $r d g B$ (retinal-degeneration$B$ ) mutations result in light-induced, age-dependent degenera- tion of photoreceptors; this morphological deterioration tends to be specific for the "outer" photoreceptors in each ommatidium (Harris and Stark, 1977), called R1-6. In flies expressing the allele we used, $r d g B^{\mathrm{Ks} 222}$, the "inner" photoreceptors in each facet (R7-8) and accessory cells of the eye are not affected (Stark and Sapp, 1987). We sectioned both $r d g B^{\mathrm{Ks} 222}$ and wild-type flies during the night (ZT 18-23) at 5 and $19 \mathrm{~d}$ posteclosion. Obvious deterioration of eye staining was seen in 19-d-old $r d g B^{\mathrm{KS} 222}$ adults $(n=4)$, unlike the wild-type $(n=1)$, for which aging had no apparent effect on per protein staining (data not shown). In the older $r d g B^{\mathrm{Ks} 222}$ flies, staining of the R7 and R8 nuclei was only slightly diminished, compared to 5-d-old mutant individuals $(n=3)$ or wild-types $(n=2)$. The results of this "genetic dissection" confirmed the hypothesis that the per protein in the eyes is in the photoreceptor cells.

Experiments on the photoreceptor mutants norp $A^{\mathrm{P} 24}$, norp $A^{\mathrm{P} 41}$, and ninaE $E^{\mathrm{O} 17}$ were undertaken to study the effects of lightmediated potentials on per protein staining. The former $2 \mathrm{mu}-$ tants are physiologically unresponsive to light in all their external photoreceptors, resulting in animals that are blind in terms of standard visually mediated behaviors (Pak, 1979; Bloomquist et al., 1988). The spatial distribution and intensities of staining in the norp $A^{\mathrm{P} 41}$ and norp $A^{\mathrm{P} 24}$ mutants-sectioned late in the night-were found to be comparable to the wild-type immunoreactivities at this time. For results obtained from a pair of flies expressing the former norp $A$ mutation, see Figure 6. Another pair of norp $A^{\mathrm{P} 41}$ adults was processed at ca. ZT 21 and ZT 8 , yielding the same staining results as depicted in Figure $6, A, B-$ strong and very weak staining, respectively. For norp $A^{\mathrm{P} 24}$, 5 adults were sectioned between ZT 20-22, and 1 was sectioned at ZT 8; the staining results (not shown) were the same as just noted.

A partially blind ninaE (neither-inactivation-nor-after-potential-E) mutant was similarly applied. This gene codes for the fly's major visual pigment, rhodopsin \#1, expressed in photoreceptors R1-6 (review: Montell et al., 1988); the absence of 


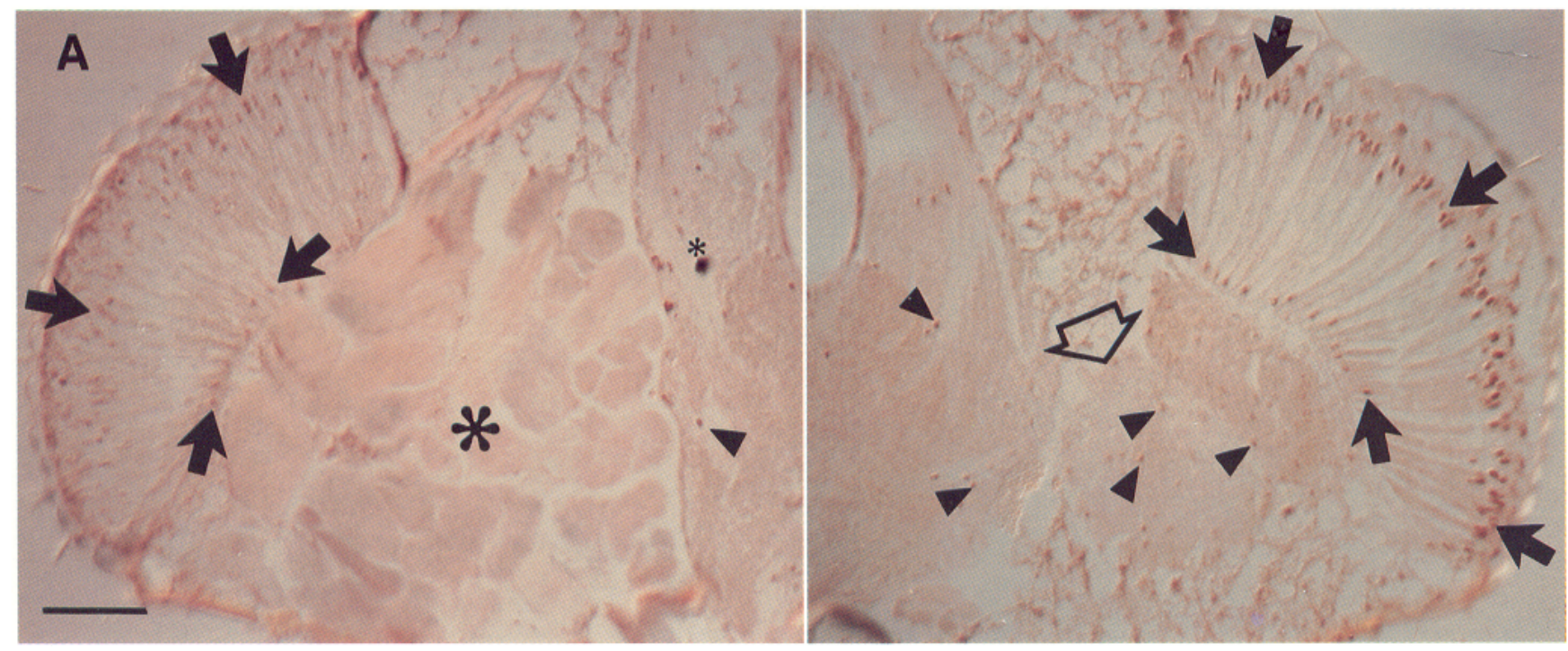

B disco photoreceptors - LD

D disco photoreceptors-DD
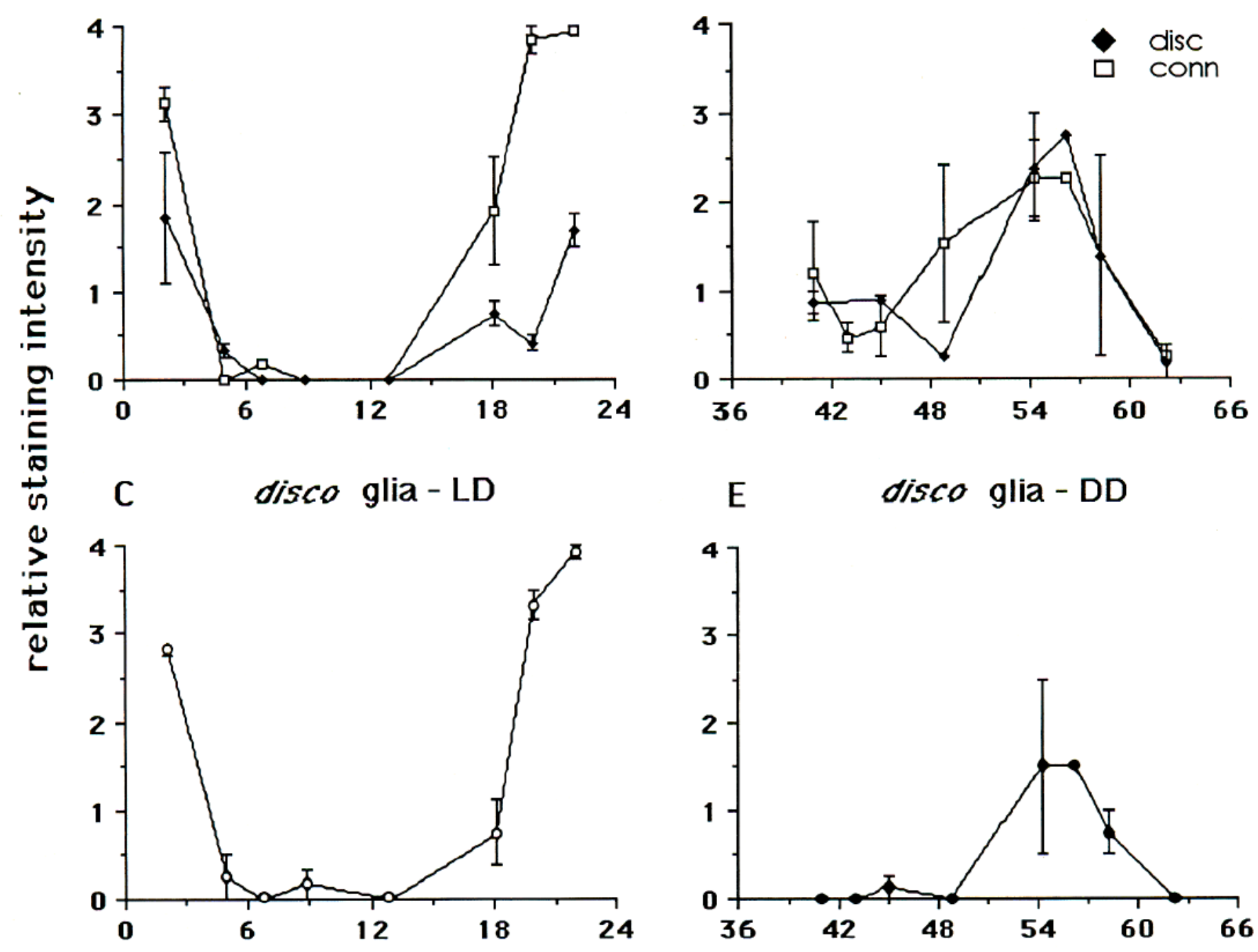

E disco glia-DD

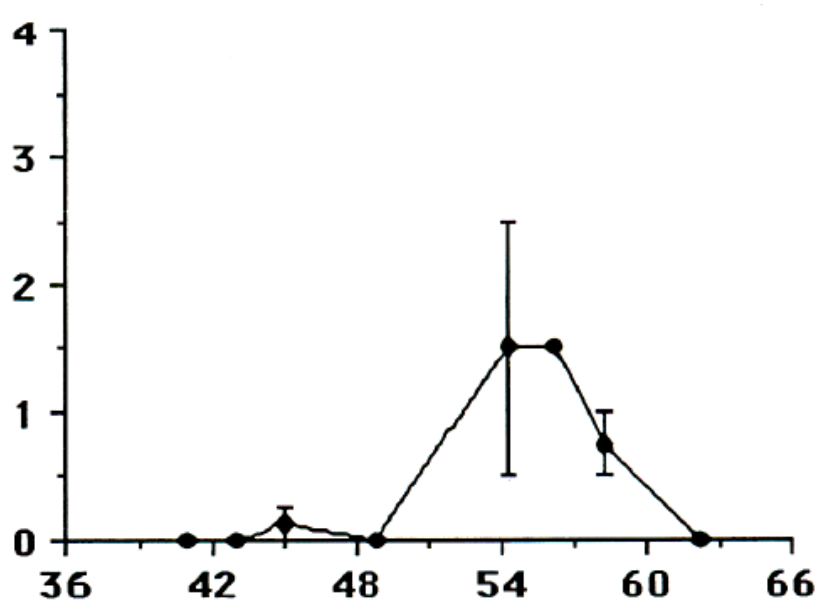

Zeitgeber time 
rhodopsin \#1 in ninaE ${ }^{\mathrm{O} 17}$, a null mutant (O'Tousa et al., 1985), causes these outer cells in each eye facet to be unresponsive to light. Four of these mutant flies were sectioned at ZT 21, and one was sectioned at ZT 9. There was strong staining at the former time and little or none at the latter (not shown; cf. Figs. $1,6)$.

These experiments, using the 3 mutants with impaired visual function, also indicated that the intensities of per immunoreactivity in the neurons, photoreceptors, and glial cells of these visual mutants cycled (in $I D$ ) with phases roughly like that of wild-type. Thus, elicitation of the basic expression and the daily oscillations of per protein in LD are independent of the normal pathway for photoreceptor excitation. Also, the normal brain neuronal staining in norp $A$ adults may correlate with the fact that these blind flies respond to LD cues by exhibiting strong circadian rhythms of locomotor activity in subsequent darkness (Dushay et al., 1989).

Mutant disconnected (disco) flies have eyes unconnected to their optic lobes (Steller et al., 1987) and are largely arrhythmic in free-running conditions (Dushay et al., 1989). The absence of normal eye-brain connections in this mutant results in the disappearance of the deep-pseudo-pupil, or DPP (see discussion in Dushay et al., 1989); this morphological phenotype can occur on both sides of the brain ( $\mathrm{DPP}^{-}$) or on one side only ( $\mathrm{DPP}^{+/-}$) in a given disco adult; the space between the eye and the central brain, normally occupied by the optic ganglia, is filled instead with disorganized muscle tissue and/or unidentifiable structures (Steller et al., 1987). In the rare disco adults with one connected $\left(\mathrm{DPP}^{+}\right)$eye, some anatomical disorganization of the visual system is still apparent; such flies - as do their thoroughly DPP- sublings - perform poorly in tests of behavioral responses to visual stimuli, and they are largely arrhythmic in their locomotor activity (Dushay et al., 1989). DPP $+/-$ individuals were studied to determine if neuronal pathways between the eye and the brain are necessary for expression and cycling of the per protein.

Staining of the photoreceptors in disco mutants was found to be reasonably robust and cycled in LD as well as in DD (Fig. 7), with time courses like those observed in wild-type (Figs. 2, 5 ). The connected eyes of disco mutants stained with intensities similar to wild-type, while the staining in disconnected eyes was somewhat less intense (Fig. 7). Rather strong glial staining (Fig. 2) was present throughout the brains of disco adults and seemed to cycle normally. It is important to note (as implied by the plots in Fig. 7) that the brain neurons which usually express per were poorly detectable in disco by application of this antibody:
Only about $15 \%$ of these mutant adults had a small number of lateral neurons staining in their usual locations; another $30 \%$ of the disco individuals examined contained a few per-expressing cells that were apparently CNS neurons, but these were scattered in ectopic (nonlateral) regions of the brain.

\section{P-element mediated transformants}

Flies with various fragments of the per gene transformcd into a per ${ }^{01}$ background were sectioned and stained with the anti-per reagent. The transformed types $14.6: 21,8.0: 4,13.2: 34$, and y w; 13.2:34/CyO; 13.2:2/13.2:2 (see Materials and Methods) were compared to one another and to wild-type in LD.

Flies transformed with a single copy of the 14.6 or the $8.0 \mathrm{~kb}$ per-DNA fragments frequently exhibit rhythmic adult behavior, though such rhythms are not like those of wild-type (Zehring et al., 1984; Hamblen et al., 1986; Yu et al., 1987a, b). About twothirds of the 14.6:21 flies are strongly rhythmic, exhibiting periods of $27-28 \mathrm{hr}$, whereas about half the flies from the 8.0:4 strain are weakly rhythmic, with ca. 24-25 hr periods (e.g., Zehring et al., 1984).

Several 14.6:21 adults were monitored, and both rhythmic and arrhythmic individuals were subsequently sectioned in an attempt to correlate rhythmicity with per protein staining in these transformed flics. The ovcrall staining intensitics in these animals were quite low. A modest correlation could be inferred between rhythmicity and staining, in that the 3 arrhythmic individuals were not immunoreactive (Table 1), although 2 of the 7 rhythmic cases were also unstained. After monitoring and subsequently sectioning transformed 8.0:4 flies, per immunoreactivity was not detected in any of the 9 individuals examined, though 5 had been weakly rhythmic behaviorally (Table 1).

Most 13.2:34-transformed individuals are strongly rhythmic; their periods are 0.5-1 hr longer than normal (Citri et al., 1987; Yu et al., 1987a). A low level of staining was found consistently in the 13.2:34's (Table 1), which may be associated with the slightly slow clock running in these transformants (see Discussion). However, no correlation was found between the intensity of per protein staining in the 13.2:34 flies and the presence or absence of rhythmicity (Table 1 ).

Flics were constructed to carry multiple doses of $p e r^{+}$DNA: one copy on the $X$ chromosome in the gene's normal location, plus 3 transduced $13.2 \mathrm{~kb}$ inserts [one dose heterozygous on chromosome 2, plus 2 doses homozygous on chromosome 3 (see Materials and Methods)]. Behavioral tests showed that these "13.2 × 3 " transformants exhibited strong rhythms of locomotor activity (Table 1), with shorter than normal periods (cf.

\footnotetext{
Figure 7. LD and DD cycling of the per protein in a disconnected mutant. Mutant disco individuals with one eye disconnected and the other connected (see text) were chosen as described by Dushay et al. (1989) and used in immunohistochemical experiments involving the anti-S reagent. $A$, This disco ${ }^{2}$ fly was sectioned and stained late in the night (ZT 22) in an LD cycle; the large asterisk marks the disorganized optic lobes of the disconnected (left) side, and the large open arrow points to an optic lobe neuropil on the connected (right) side. The photoreceptor nuclei (large filled arrows) were stained in both eyes, though less intensely on the disconnected (left) side. Glial staining (arrowheads) was normal in the optic lobes of the connected side and in the central brain. Stained lateral neurons were rarely observed in disco flies. Small asterisk, debris. Scale bar, $50 \mu \mathrm{m}$. $B-E$, Time courses of the changing staining intensities of the per protein in disco photoreceptors and glia in LD cycles ( $B, C)$, and after $40-$ $60 \mathrm{hr}$ in constant darkness, postentrainment $(D, E)$. Separate scores were recorded for photoreceptors of connected (conn) and disconnected (disc) eyes, before plotting the data in $B$ and $D$. Each time point in the 4 panels represents scoring of 2-3 flies, averaged as described in Materials and Methods. For $B$, in which both the disc and conn curves reach troughs at zero staining, the filled diamonds plotted at ZT 9 and 13 represent overlapping points on the 2 curves. The horizontal time bars are explained in Figures 2 and 5 . After the DD scores were plotted $(D, E)$, extrapolations were performed as in Figure 5 and indicated that the free-running rhythms of the per protein in disco photoreceptors and glia had periods of ca. $24 \mathrm{hr}$. Panel $E$ shows that glial staining was detected in this mutant after 54-58 hr in DD, in contrast to the lack of staining in wild-type glia under these conditions (see Fig. 5).
} 
Table 1. Behavioral rhythms and per protein staining of per transformants

\begin{tabular}{|c|c|c|c|c|c|}
\hline \multirow[b]{2}{*}{ Genotype } & \multirow{2}{*}{$\begin{array}{l}\text { Mean period } \\
\text { for rhythmic } \\
\text { flies } \\
(\mathrm{hr} \pm \mathrm{SEM})\end{array}$} & \multicolumn{4}{|c|}{$\begin{array}{l}\text { Relative staining intensity in brain neurons } \\
\text { in flies whose behavior was }\end{array}$} \\
\hline & & Rhythmic $(n)$ & & Arrhythmic (n) & Not tested $(n)$ \\
\hline "13.2" transformant & $24.8 \pm 0.2$ & $1.0 \pm 0.2$ & (5) & $1.8 \pm 1.3(2)$ & $1.7 \pm 0.4(5)$ \\
\hline Male & $23.0 \pm 0$ & (see footnote) & (3) & - & - \\
\hline Female & $23.5 \pm 0$ & & (3) & - & - \\
\hline “14.6" transformant & $27.9 \pm 0.5$ & $0.4 \pm 0.1$ & (7) & (3) & - \\
\hline “8.0" transformant & $24.4 \pm 0.5$ & 0 & (5) & (4) & 0 \\
\hline per ${ }^{+}$control & - & - & & - & $2.4 \pm 0.4(9)$ \\
\hline
\end{tabular}

Flies carrying the transduced per $^{+}$DNA fragments (against a genetic background of per ${ }^{\text {I }}$ ) had their locomotor activity monitored in DD, after LD entrainment (see Materials and Methods and Hamblen et al., 1986, for further details). The adults were then subjected to a few additional days of $L D$, before being frozen, sectioned, and stained using the antiper antibody. Flies were frozen late in the night (ZT 1930-2230). For 3 of the genotypes, some additional animals were processed without having been monitored for behavioral rhythmicity (right-most column); these flies had been in LD conditions and were frozen between ZT 2030 and 2300 . Details about the specific transformed strains used are in Materials and Methods (also see Zehring et al., 1984; Citri et al., 1987). The per flies were from a Canton-S wild-type or from $y w$ or $r y$ strains; these controls were done independently of those in Figures 2 and 4 , and Table 2 . All of the transformed and control flies were males, except for 3 of the "13.2 $\times 3$ " animals (a transformant strain carrying 3 copies of the transduced $13.2 \mathrm{~kb} \mathrm{per}$ insert; see Materials and Methods). Assessments of rhythmicity, including best estimates of circadian periods, were accomplished by $\chi^{2}$ periodogram analyses (Hamblen et al., 1986). Scorings of staining intensities were done blind (with regard to genotype and behavioral rhythmicity), using the "0-to-4" scale described in Materials and Methods and Results. These numbers led to computations of mean staining scores ( \pm SEM), which are tabulated here for the lateral brain neurons ( $c f$. Fig. 1, $A, C$ ). The scores for photoreceptors and glia were generally correlated with those for the neurons, though there were some exceptions: For example, the glia were uniformly 0 in the 13.2 transformants, and both the eyes and glia were 0 in the 14.6 transformants. For the "13.2 $\times 3$ " transformants, staining intensities in the lateral brain neurons were appreciably greater than in $3 \mathrm{per}^{+}$controls sectioned in parallel; however, the wild-type staining intensities for this experiment were unusually low, so these results were not tabulated. The numbers of flies $(n)$ are in parentheses.

Smith and Konopka, 1982). The intensities of per protein staining in these flies were higher than that seen in the $p e r^{+}$controls done in parallel (see Table 1 footnote).

\section{Alterations of light and dark conditions}

In wild-type culture of Drosophila that are transferred into DD after exposure to LD cycles, there are peaks of eclosion occurring ca. $24 \mathrm{hr}$ apart; in contrast, cultures transferred into constant light (LL) do not eclose rhythmically (review: Saunders, 1982). It has also been shown that adult flies, entrained by LD then put into DD, exhibit rhythms of locomotor activity which, upon thcir subscquent transfer into LL, degenerate into arrhythmicity (Konopka et al., 1989). We raised 20 wild-type flies in LL, then monitored their locomotor activity in this condition (see Materials and Methods)-an experiment which seems not to have been done previously with Drosophila. Only one fly was weakly rhythmic (by $\chi^{2}$ periodogram analysis, cf. Hamblen et al., 1986), and its period was barely circadian, i.e., $21 \mathrm{hr}$. The largely arrhythmic behavior of wild-type flies in constant light is essentially a phenocopy of the per ${ }^{0}$ mutants, since $3-5 \%$ of adults expressing per-null alleles are weakly rhythmic by these statistical methods (Hamblen et al., 1986).

In immunohistochemical experiments carried out against a background of conditions similar to those just described, wildtype flies transferred into LL after a few days of entrainment showed very weak staining of the per protein (Table 2, line 3). Animals raised from embryos in LL also exhibited, as adults, essentially no per immunoreactivity (Table 2, line 6).

One light-to-dark transition was sufficient to elicit robust per protein staining (Table 2, line 7). In the analogous behavioral experiments, flies subjected to one $\mathrm{LL} \rightarrow \mathrm{DD}$ transition have exhibited reasonably robust free-running activity rhythms (e.g., Petersen et al., 1988). Further behavioral tests of this kind (see Materials and Methods) showed that, of $23 \mathrm{per}^{+}$flies reared in LL (using the same light intensity as in the immunohistochemical experiments), then transferred to DD, 19 were significantly rhythmic (mean $\tau \pm \mathrm{SEM}, 23.8 \pm 0.2 \mathrm{hr}$ ).

Flies raised from embryos in DD are poorly rhythmic: Only about $25 \%$ of them have exhibited weak circadian rhythms; about $10 \%$ showed very short-period rhythms; another $10 \%$ exhibited weak long-period behavioral cycles; and about $50 \%$ were completely arrhythmic (Dowse and Ringo, 1989). Staining for the per protein in wild-type flies raised in DD was usually faint in the photoreceptors, but it was relatively strong in the brain neurons (Table 2, line 4 ). These immunohistochemical results superficially do not seem to correlate with the behavioral ones (however, see Discussion).

One $12 \mathrm{hr}$ cycle of light confers rhythmicity to insects that are raised in DD (review: Saunders 1982), although this has apparently not been documented for Drosophila. An analogous result was found immunohistochemically: DD-reared flies, given $12 \mathrm{hr}$ of light as adults, exhibited more intense stainingespecially in the photoreceptors-than seen in flies with no exposure to light (Table 2 , line 5).

The effects of various light/dark regimes on the intensity of per protein staining in the thoracic gut did not correlate well with the influences of these environmental manipulations on either neuronal stainings or behavioral rhythms (data not shown). These observations included detection of staining intensities appreciably above background levels in flies raised and subsequently maintained in $\mathrm{LL}$, a condition that led to almost no per immunoreactivity in the head (Table 2 , line 6). 
Table 2. Effects of light/dark manipulations on per protein staining

\begin{tabular}{|c|c|c|c|c|c|c|c|}
\hline \multirow{2}{*}{\multicolumn{2}{|c|}{ conditions }} & & \multicolumn{5}{|c|}{ relative staining intensities } \\
\hline & & & neurons & $(n)$ & photo & recept & $s(n)$ \\
\hline 1 & $* *$ & LD & $3.0 \pm 0.3$ & (8) & 3.3 & \pm 0.2 & (9) \\
\hline 2 & $* *$ & $L D-->D D$ & $1.6 \pm 0.6$ & (6) & 2.3 & \pm 0.5 & (6) \\
\hline 3 & $\stackrel{* *}{\rightleftarrows}$ & $L D-->L L$ & $0.3 \pm 0.2$ & (6) & 0.6 & \pm 0.1 & $(6)$ \\
\hline 4 & $*$ & DD & $1.9 \pm 0.5$ & (9) & 0.6 & \pm 0.2 & $(10)$ \\
\hline 5 & ** & $D D-->L-->D D$ & $2.3 \pm 0.6$ & (6) & 1.7 & \pm 0.2 & $(7)$ \\
\hline 6 & $\stackrel{*}{*}$ & LL & $0.1 \pm 0.1$ & (8) & 0.0 & \pm 0.0 & (9) \\
\hline 7 & 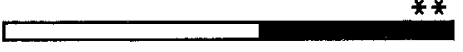 & $\mathrm{LL}-->\mathrm{DD}$ & $3.6 \pm 0.3$ & (5) & 1.2 & \pm 0.5 & $(6)$ \\
\hline
\end{tabular}

Wild-type flies were subjected to various light/dark regimes that are known to affect behavioral rhythms (see text). The experimental conditions are represented by the open and filled bars. A short stretch of filled or empty bar corresponds to $12 \mathrm{hr}$. (Thus, for example, there are $3.512 / 12 \mathrm{LD}$ cycles in line 1 .) The asterisks denote very roughly (see below) the times when flies were sectioned, with reference to the previous $L \rightarrow D$ or $D \rightarrow L$ transition. In the experiments on lines 4 and 6 , there were no such transitions; so these constant DD and constant LL flies were processed over the course of a few days, i.e., at various ages posteclosion (the widely spread out asterisks are thus meant to indicate that these animals were unsynchronized). The intensities of per protein stainings in lateral neurons and photoreceptors were scored blindly as described in Materials and Methods and Figure 2. The numbers of flies $(n)$ whose sections were scored is sometimes less for "neurons" than for "photoreceptors," because the integrity of certain sections, corresponding to the positions of the per-expressing lateral neurons ( $c f$. Fig. 1C), was occasionally not good enough to allow for careful quantitative assessment of staining intensities; in contrast, nearly any plane of section through the head allows such intensities in the compound eyes to be reliably scored. The $L D$ and the LD $\rightarrow \mathrm{DD}$ experiments were performed independently of those plotted in Figures 2 and 5 , respectively. In the LD $\rightarrow$ LL experiment (line 3), the flies were first entrained by $512 / 12 \mathrm{LD}$ cycles (though only 1.5 of these are symbolized in line 3); they were then maintained in constant light for lengths of time (43-56 hr) that put most of them in late subjective night or early subjective day - which would have yielded relatively intense stainings had this been an LD or $\mathrm{LD} \rightarrow$ DD experiment. In the $12 \mathrm{hr}$ light-pulse experiment (line 5), the flies were returned to DD for either 33-35 or 80-84 hr ( $n=3$ and 4, respectively; only the first of these durations is indicated by the asterisks); this would put the flies in late subjective night/early subjective day; hence, staining should be near its peak of intensity (see above). In the experiment involving one light-to-dark transition (line 7), the flies were again frozen and sectioned $33-35 \mathrm{hr}(n=2)$ or 80-84 hr $(n=4)$ after the environmental change.

\section{Discussion}

We have used an immunohistochemical assay for the protein encoded by the period locus of Drosophila melanogaster and found that this gene product both defines its own circadian fluctuations and influences the phase and period of these time courses. Daily fluctuations in the intensity of per protein staining describe an endogenous type of cycling, which "free-runs" in constant darkness after being entrained by light/dark cycles (Fig. 5). Like Drosophila's circadian rhythms of eclosion and locomotor activity, these protein changes are sensitive to the periodshortening effects of the pers mutation. We believe that the daily fluctuations of per immunoreactivity must be relevant to the mechanism by which this gene influences the fly's circadian clock.

This same antibody has been used to track diurnal cycling in another protein, extracted from the eye of Aplysia californica; the anti-per reagent also stained circadian pacemaker neurons in the eyes of this mollusc, as well as in another gastropod, Bulla gouldiana (Siwicki et al., 1989). The antigenic protein in Aplysia eyes was detected on Western blots as a $48 \mathrm{kDa}$ band, whose intensity fluctuated in LD cycles. The phase of this apparent rhythm was the same as that of the per protein cycling in Drosophila (Figs. 2, 4), in that peak band intensities occurred at about lights-on (Siwicki et al., 1989). Similar kinds of biochemical experiments using this antibody have not been possible in Drosophila because the anti-S reagent does not detect a perspecific protein in immunoblotting assays involving fly extracts (Siwicki et al., 1988).

The per protein has, however, been readily detectable in situ (Siwicki ct al., 1988). This has now allowed for the fluctuations of per protein staining to be monitored immunohistochemically in specific cell types within the nervous system of adult flies: (1) photoreceptors of the compound eye (Siwicki et al., 1988),
(2) certain neurons in the lateral protocerebral cortex (Fig. 1, $A$, $C$ ), and (3) cells that are probably neuropil glia.

The circadian changes in the per protein could be either an integral component of the fly's pacemaker mechanism or merely an element of the clock's output. Molecular circadian rhythms in the latter category include those involving vasopressin mRNA in the rat suprachiasmatic nucleus (Uhl and Reppert, 1986; Robinson et al., 1988), and a rhythm in the mRNA encoding the light-harvesting chlorophyll $\mathrm{a} / \mathrm{b}(\mathrm{Cab})$ protein in plants (Nagy et al., 1988; Taylor, 1989). We would predict that a mutation in the $C a b$ gene should not alter the plants' other circadian rhythms, just as a severe mutation within the rat gene encoding vasopressin allows for readily apparent (albeit somewhat weakened) sleep rhythms (Brown and Nunez, 1989). In contrast, per mutations cause dramatic changes in several of the fly's biological rhythms (reviews: Konopka, 1987; Hall and Rosbash, 1988; Young et al., 1989), suggesting that the per protein is actually a gear in Drosophila's circadian clock.

Of the several cell types that showed anti-per labeling, the neurons in the lateral protocerebrum (Fig. $1 C$ ) seem to be the best candidates for the anatomical substrates of the fly's circadian pacemaker. As discussed in our previous report (Siwicki et al., 1988), some of the lines of evidence supporting this hypothesis are (1) genetic mosaic studies implicating tissues in the fly's head as the site of per's influence on circadian locomotor activity rhythms (Konopka et al., 1983); (2) results of mutant and lesioning studies indicating that dipteran circadian pacemakers are not likely to be in the eyes or optic lobes (reviewed by Hall and Kyriacou, 1990; though see Hofbauer and Buchner, 1989); and (3) staining experiments showing that the lateral neurons were the only anti-S-labeled structures in the heads of late pupae (Siwicki et al., 1988), which do have functional circadian pacemakers (Saunders, 1982). These arguments are now augmented by (4) the poor detectability of the lateral neurons 
(which could mean that they are usually absent) in the arrhythmic disco mutant (Fig. 7; see also Dushay et al., 1989) and (5) the reasonably good correlations of staining intensities in these brain cells with the "strengths" and/or periodicities of circadian behavioral rhythms in the per ${ }^{\mathrm{L} 1}$ mutant or in per transformants (see below).

In this regard, $p e r^{\mathrm{L} 1}$ adults exhibited weak staining and a lowamplitude rhythm in LD (Fig. 3). Whereas it was difficult to compare the time courses of the per ${ }^{\mathrm{L}}$ and $\mathrm{per}^{+}$protein fluctuations, the weak expression of per in this long-period mutant fits loosely with schemes relating quantities of the gene's product with period lengths: the lower these levels, the longer the cycle durations (Smith and Konopka, 1982; Coté and Brody, 1986; Baylies et al., 1987).

The overall pattern of results from behaviorally monitoring, then sectioning and staining, per transformants was consistent with the levels of immunoreactivity seen in the long-period mutant. Thus, more intense (peak) staining levels in transformed adults tended to correlate with stronger and/or shorter-period circadian rhythms (Table 1): The "13.2" transformant type has the most nearly normal rhythms of locomotor activity (Citri et al., 1987; Yu et al., 1987b) and showed the strongest stainings. The "14.6" type has rhythms with much longer periods, though these flies' rest/activity cycles are self-evident in plots of their locomotor behavior (Zehring et al., 1984; Hamblen et al., 1986); their anti-per-mediated stainings were faint but detectable (Table 1). Finally, the " 8.0 " transformants, despite their near-normal periodicities (24-25 hr; Zehring et al., 1984), exhibit adult behavioral rhythms that are very weak (often lacking clearly discernible differences between activity peaks and troughs), and they sometimes degrade into arrhythmicity (Zehring et al., 1984). Examples of these 8.0-mediated activity plots are in Hamblen et al. (1986) and Yu et al. (1987b). We believe that at least the rhythmic 8.0-transformants contained functional per protein; however, staining was not revealed in these experiments (Table 1), probably because the protein level was below the limit of detection with this antibody. Indeed, some adult tissues that do express per, such as ovaries and ring gland (Liu et al., 1988; Saez and Young, 1988), are not stained by application of the anti-S reagent (Siwicki et al., 1988). In this regard, it is notable that the 8.0 transformants express extremely subnormal levels of per mRNA (Hamblen et al., 1986).

The most telling case so far is pers. The circadian fluctuation in this mutant's protein in DD had a shorter than normal period (Fig. 5). Even in LD (where the periodicity is imposed by the $24 \mathrm{hr}$ environmental cycle), the time course of changes in the $p e r^{s}$ protein differed from wild-type. The neuronal staining in this mutant decayed earlier than in $\mathrm{per}^{+}$during the day (Fig. $4 A$ ), and the photoreceptor and glial stainings rose faster and peaked sooner during the night (Fig. 4, B, C). This effect of per in a sense parallels the mutant's behavior in $12 \mathrm{hr}$ light/12 hr dark cycles: Whereas the periods of such activity rhythms are "forced" to be $24 \mathrm{hr}$, pers locomotor activity peaks occur substantially earlier than those of wild-type flies (M. HamblenCoyle and D. A. Wheeler, unpublished observation).

It has been inferred, though never demonstrated, that the $p^{\mathrm{s}}$ mutant is hyperactive for the function of this gene or its product (Smith and Konopka, 1982). More specifically, pers is hypothesized to bc about 35 times more active than the normal allele, given the degree of period shortening effected by this mutation (Coté and Brody, 1986). Since there are apparently normal levels of per transcript in this mutant (Bargiello and Young, 1984;
Hardin et al., 1990), and because pers is accounted for by a missense mutation (Baylies et al., 1987; Yu et al., 1987b), it would seem as if the hyperactivity in question occurs at the level of a given protein molecule. Consistent with this inference is the fact that the peak levels of pers protein stainings determined in the current experiments are not elevated above those of wildtype (Figs. 4, 5).

Other parallels between behavioral rhythms and per expression were revealed by manipulating the environmental conditions (Table 2). For most of the light/dark regimes tested, the effects on per protein staining were consistent with the behavioral effects of the same conditions (Petersen et al., 1988; Konopka et al., 1989). The only ambiguous case was that of flies raised in constant darkness (DD). Although about half of the animals always kept in DD have been reported to be arrhythmic (Dowse and Ringo, 1989), we found reasonably strong per staining in the lateral brain neurons of every such fly we examined immunohistochemically (Table 2). One interpretation of these results is that per expression in these neurons is necessary but not sufficient for rhythmicity. Some additional factor (e.g., cxposurc to light) might be required to "start the clock." Another possibility which is consistent with these findings is that the protein cycles independently in the individual per-expressing neurons of DD flies and that the effect of an environmental transition is not so much to start the clock as to synchronize the separate cellular pacemakers.

We could not determine whether the per protein cycled in DD flies since, in the absence of entrainment cues, any putative rhythms in the individual adults were likely to have been unsynchronized. Consider, though, the possibility that the level of per product simply does not fluctuate appreciably in the neurons of flies kept in constant darkness (even within a given cell, let alone in synchrony among cells). This would imply that it is not sufficient for the relevant neurons merely to contain per product; for behavioral rhythmicity to be strong, the protein would also have to exhibit robust oscillations in its abundance. The effects of DD (Dowse and Ringo, 1989) could therefore approach in severity those of the opposite kind of treatment: the fact that putting the flies into, or always keeping them in, constant light leads to arrhythmicity and little or no per neuronal staining (Konopka et al., 1989, and Table 2 of this report).

Our studies of visual mutants have shed new light on several aspects of the expression and function of per. The case of disco, with its nonstaining of the lateral brain neurons, not only suggests that these cells are the fly's circadian pacemaker structure (see above), but is also informative with regard to per immunoreactivity in the photoreceptors. The circadian fluctuations of per protein staining appeared to be normal, in disco eyes, when these structures were either connected or disconnected from the brain (Fig. 7). Recall that almost every disco adult tested has been arrhythmic and that they exhibited such locomotor activity rhythm defects irrespective of whether their cycs were connected, via optic ganglia, to the brain (Dushay et al., 1989). Thus, there is a dissociation of the behavioral and photoreceptor staining rhythms. Nonetheless, this is consistent with several lines of evidence indicating that the presence or function of the fly's external eyes is not necessary for its behavioral circadian rhythms (review: Hall and Kyriacou, 1990).

It is possible that the Drosophila eye may have an autonomous rhythm which would be influenced by per protein oscillations. There is, in fact, an apparent rhythmicity associated with rhabdomere turnover in these photoreceptors (Stark et al., 1988), 
which is probably not related to the fly's behavioral circadian cycles. In this respect, it is noteworthy that the lateral neuron and photoreceptor CNS staining rhythms have slightly different phases in LD (Figs. 2, 4). It is still, however, possible that the eye rhythm is controlled by output from the brain. Thus, there could be a functional central pacemaker in disco which might regulate the photoreceptor rhythm by hormonal signals (not requiring the eye to be connected to the brain via efferents in the optic ganglia). Yet, other output pathways from the CNS, those controlling locomotor activity, would be disrupted by disco mutations, accounting for their effects on behavioral rhythms (Dushay et al., 1989).

Whether the circadian pacemaker regulating the per protein fluctuations in photoreceptors is endogenous to the eye or is in the central brain, its entrainment by light is independent of the normal pathways of photoreceptor excitation. This is because photoreceptor cells that lack rhodopsin, in a ninaE mutant (O'Tousa et al., 1985), or are "phototransduction-null" for other reasons, in norpA (Bloomquist et al., 1988), nevertheless had normal day versus night differences in per staining. Thus, these cells (Fig. 6) receive and process the signals necessary to turn on per cycling (Table 2) by an as yet unidentified transduction pathway.

In summary, we infer that there is a circadian rhythm in the protein product of the period gene. The changing levels (or quality) of the per protein are likely to be an important aspect of the mechanism by which this gene regulates the fly's circadian rhythms. Since the qualitatively altered protein encoded by the pers allele (Baylies et al., 1987; Yu et al., 1987b) changes the temporal modulation of this gene's product, we hypothesize that some kind of feedback loop is involved in the fly's circadian clock. That is, the activity of the per protein directly or indirectly influences its own abundance. This effect probably involves oscillations in the rate of per protein synthesis, given the recent discovery of a robust circadian rhythm in the levels of the 4.5 kb RNA transcribed from this gene (Hardin et al., 1990). The phase of this mRNA abundance rhythm is approximately $6 \mathrm{hr}$ in advance of the per protein rhythm (Hardin et al., 1990). Thus, it is likely that the oscillations in staining intensity reflect changes in the quantity, rather than the quality (e.g., epitope accessibility), of the protein. Other elements of the loop, and hence of this molecular circadian clock, may include factors regulating the turnover of per's transcript, the rate of degradation of the protein, or both.

By analogy to the rhythmic changes in the membrane potential of circadian pacemaker neurons in the eye of Bulla (McMahon et al., 1984), we might expect physiological oscillations in Drosophila's per-expressing cells to be part of this feedback loop. The presence of a per-like antigen in these molluscan pacemaker neurons (Siwicki et al., 1989) offers interesting prospects for identifying the links between the molecular rhythms in the per or per-related gene products and the neurophysiological properties of circadian pacemaker neurons.

\section{References}

Bargiello TA, Young, MW (1984) Molecular genetics of a biological clock in Drosophila. Proc Natl Acad Sci USA 81:2142-2146.

Baylies MK, Bargiello TA, Jackson FR, Young MW (1987) Changes in abundance or structure of the per gene product can alter periodicity of the Drosophila clock. Nature 326:390-392.

Besharse JC, Iuvone PM (1983) Circadian clock in Xenopus eye controlling retinal serotonin $N$-acetyltransferase. Nature 305:133-135.
Bloomquist BT, Shortridge RD, Schneuwly S, Perdew M, Montell C, Steller H, Rubin GM, Pak WL (1988) Isolation of a putative phospholipase $C$ gene of Drosophila, norpA, and its role in phototransduction. Cell 54:723-733

Brown MH, Nunez AA (1989) Vasopressin-deficient rats show a reduced amplitude of the circadian sleep rhythm. Physiol Behav 46: $759-762$.

Citri Y, Colot HV, Jacquier AC, Yu Q, Hall JC, Baltimore D, Rosbash $M$ (1987) A family of unusually spliced and biologically active transcripts is encoded by a Drosophila clock gene. Nature 326:42-47.

Coté GG, Brody S (1986) Circadian rhythms in Drosophila melanogaster: analysis of period as a function of gene dosage at the per (period) locus. J Theor Biol 121:487-503.

Dowse HB, Ringo JM (1989) Rearing Drosophila in constant darkness produces phenocopies of period circadian clock mutants. Physiol Zool 62:785-803.

Dushay MS, Rosbash M, Hall JC (1989) The disconnected visual system mutations in Drosophila melanogaster drastically disrupt circadian rhythms. J Biol Rhythms 4:1-27.

Hall JC, Kyriacou CP (1990) Genetics of biological rhythms in Drosophila. Adv Insect Physiol 22:221-298.

Hall JC, Rosbash M (1988) Mutations and molecules influencing biological rhythms. Annu Rev. Neurosci 11:373-393.

Hamblen M, Zehring WA, Kyriacou CP, Reddy P, Yu Q, Wheeler DA, Zwiebel LJ, Konopka RJ, Rosbash M, Hall JC (1986) Germ-line transformation involving DNA from the period locus in Drosophila melanogaster: overlapping genomic fragments that restore circadian and ultradian rhythmicity to per and per- mutants. J Neurogenet 3 : 249-291.

Hardin PE, Hall JC, Rosbash M (1990) Feedback of the Drosophila period gene product on circadian cycling of its messenger RNA levels. Nature 343:536-540.

Harris WA, Stark WS (1977) Hereditary retinal degeneration in Drosophila melanogaster: a mutant defect associated with the phototransduction process. J Gen Physiol 69:261-292.

Hofbauer A, Buchner E (1989) Does Drosophila have seven eyes? Naturwissenschaften 76:335-336.

Hoyle G (1986) Glial cells of an insect ganglion. J Comp Neurol 246: $85-103$.

Johnson CH, Hastings JW (1986) The elusive mechanism of the circadian clock. Am Sci 74:29-36.

Konopka RJ (1987) Genetics of biological rhythms in Drosophila. Annu Rev Genet 21:227-236.

Konopka RI, Benzer S (1971) Clock mutants of Drosophila melanogaster. Proc Natl Acad Sci USA 68:2112-2116.

Konopka RJ, Wells S, Lee T (1983) Mosaic analysis of a Drosophila clock mutant. Mol Gen Genet 190:284-288.

Konopka RJ, Pittendrigh C, Orr D (1989) Reciprocal behavior associated with altered homeostasis and photosensitivity of Drosophila clock mutants. J Neurogenet $6: 1-10$.

Liu X, Lorenz L, Yu Q, Hall JC, Rosbash M (1988) Spatial and temporal expression of the period gene in Drosophila melanogaster. Genes Dev 2:228-238.

McMahon DG, Wallace SF, Block GD (1984) Cellular analysis of the Bulla ocular circadian pacemaker system. II. Neurophysiological basis of circadian rhythmicity. J Comp Physiol 155:379-385.

Montell C, Mismer D, Fortini ME, Zuker CS, Rubin GM (1988) Identification and expression of Drosophila phototransduction genes. In: Molecular biology of the eye: genes vision, and ocular disease (Piatigorsky J, Shinohara T, Zelenka PS, eds), pp 277-291. New York: Liss.

Nagy F, Kay SA, Chua N-H (1988) A circadian clock regulates transcription of the wheat Cab-1 gene. Genes Dev 2:376-382.

O'Tousa JE, Baehr W, Martin RL, Hirsh J, Pak WL, Applebury ML (1985) The Drosophila ninaE gene encodes an opsin. Cell 40:839850

Pak WL (1979) Study of photoreceptor function using Drosophila mutants. In: Neurogenetics: genetic approaches to the nervous system (Breakefield XO, ed), pp 67-99. New York: Elsevier/North Holland.

Petersen G, Hall JC, Rosbash M (1988) The period gene of Drosophila carries species-specific behavioral instructions. EMBO J 7:3939-3947.

Robinson BG, Frim DM, Schwartz WJ, Majzoub JA (1988) Vasopressin mRNA in the suprachiasmatic nuclei: daily regulation of polyadenylate tail length. Science 241:342-344.

Saez L, Young MW (1988) In situ localization of the per clock protein 
during development of Drosophila melanogaster. Mol Cell Biol 8: 5378-5385.

Saunders DS (1982) Insect clocks, 2nd ed. Oxford, UK: Pergamon.

Siwicki KK, Eastman C, Petersen G, Rosbash M, Hall JC (1988) Antibodies to the period gene product of Drosophila reveal diverse tissue distribution and rhythmic changes in the visual system. Neuron 1: 141-150.

Siwicki KK, Strack S, Rosbash M, Hall JC, Jacklet JW (1989) An antibody to the Drosophila period protein recognizes circadian pacemaker neurons in Aplysia and Bulla. Neuron 3:51-58.

Smith RF, Konopka RJ (1982) Effects of dosage alterations at the per locus on the period of the circadian clock of Drosophila. Mol Gen Genet 185:30-36.

Stark WS, Sapp R (1987) Ultrastructure of the retina of Drosophila melanogaster, the mutant ora (outer rhabdomeres absent) and its inhibition of degeneration in $r d g B$ (retinal degeneration-B). J Neurogenet 4:227-240.

Stark WS, Sapp R, Schilly D (1988) Rhabdomere turnover and rhodopsin cycle: maintenance of retinula cells in Drosophila melanogaster. J Neurocytol 17:499-509.

Steller H, Fischbach K, Rubin GM (1987) disconnected: a locus required for neuronal pathway formation in the visual system of Drosophila. Cell 50:1139-1153.

Takahashi JS, Hamm H, Menaker M (1980) Circadian rhythms of melatonin release from individual superfused chicken pineal glands in vitro. Proc Natl Acad Sci USA 77:2319-2322.

Taylor WC (1989) Transcriptional regulation by a circadian rhythm. Plant Cell 1:259-264.

Uhl GR, Reppert SM (1986) Suprachiasmatic nucleus vasopressin messenger RNA: circadian variation in normal and Brattleboro rats. Science 232:390-393.

Wigglesworth VB (1959) The histology of the nervous system of an inscct, Rhodnius prolixus (Hemiptera). II. The central ganglia. Q J Microsc Sci 100:299-313.

Young MW, Bargiello TA, Baylies MK, Saez L, Spray DC (1989) Molecular biology of the Drosophila clock. In: Neuronal and cellular oscillators (Jacklet JW, ed), pp 529-542. New York: Marcel Dekker.

Yu Q, Colot HV, Kyriacou CP, Hall JC, Rosbash M (1987a) Behaviour modification by in vitro mutagenesis of a variable region within the period gene of Drosophila. Nature 326:765-769.

Yu Q, Jacquier AC, Colot HV, Citri Y, Hamblen M, Hall JC, Rosbash M (1987b) Molecular mapping of point mutations in the period gene that stop or speed up biological clocks in Drosophila melanogaster. Proc Natl Acad Sci USA 84:784--788.

Zehring WA, Wheeler DA, Reddy P, Konopka RJ, Kyriacou CP, Rosbash M, Hall JC (1984) P-element transformation with period locus DNA restores rhythmicity to mutant, arrhythmic Drosophila melanogaster. Cell 39:369-376. 